\title{
NTRK1 and NTRK2 receptors facilitate follicle assembly and early follicular development in the mouse ovary
}

\author{
Bredford Kerr, Cecilia Garcia-Rudaz, Mauricio Dorfman, Alfonso Paredes ${ }^{1}$ and Sergio R Ojeda \\ Division of Neuroscience, Oregon National Primate Research Center/Oregon Health and Science University, \\ 505 Northwest 185th Avenue, Beaverton, Oregon 97006, USA and ${ }^{1}$ Laboratory of Neurobiochemistry, Faculty of \\ Chemistry and Pharmaceutical Sciences, Universidad de Chile, Sergio Livingstone Polhammer 1007, Independencia, \\ Santiago 6640750, Chile
}

Correspondence should be addressed to S R Ojeda; Email: ojedas@ohsu.edu

B Kerr and C Garcia-Rudaz contributed equally to this work

\begin{abstract}
Recent studies have demonstrated that neurotrophins (NTs) and their NTRK tyrosine kinase receptors, thought to be exclusively required for the development of the nervous system, are also involved in controlling ovarian development. Here, we show that primordial follicle formation is decreased in the absence of nerve growth factor (NGF) or its receptor NTRK1, and in the absence of NTRK2, the receptor for neurotrophin-4 (NTF4) and brain-derived neurotrophic factor (BDNF). This deficiency is not due to premature oocyte loss, because the ovaries of $\mathrm{Ntrk1}^{-/-}$and Ntrk2${ }^{-/-}$mice do not show an increased rate of oocyte death antedating the initiation of folliculogenesis. Moreover, exposure of NGF-deficient ovaries to NGF rescues the defect in follicular assembly, if NTRK1 receptors are present, suggesting that the absence of NTs causes a delay, and not an irretrievable loss, of follicle formation. Both the number of secondary follicles and FSH receptor (FSHR) expression are diminished in Ntrk1- and Ntrk2-null ovaries, but not in ovaries lacking the common NT receptor NGFR. Transient exposure of wild-type ovaries to NTF4 increases Fshrgene expression and enhances the ability of the ovary to respond to FSH with formation of cyclin D2, a cell cycle protein mediating the proliferative actions of FSH in the ovary. These results indicate that both NTRK1 and NTRK2 receptors are necessary for the timely assembly of primordial follicles and for sustaining early follicular development. They also suggest that a mechanism by which NTRK2 receptors facilitate subsequent follicle development is by inducing the formation of functional FSHR.

Reproduction (2009) 138 131-140
\end{abstract}

\section{Introduction}

Much is known about the hormonal mechanisms controlling ovarian development. More recently, a major focus of attention in the field has been the identification of regulatory pathways that, operating within the ovarian microenvironment, contribute to the acquisition of ovarian reproductive competence. Within this framework, our laboratory has developed the concept that neurotrophins (NTs) and their NTRK tyrosine kinase receptors, long thought to be exclusively required for the development of the nervous system, are also involved in the control of ovarian function (reviewed in (Dissen et al. 2004)).

A role for NTs in the control of ovarian maturation was initially suggested by the finding that the developing ovary not only contains four of the known NTs (nerve growth factor, NGF; brain derived neurotrophic factor, BDNF; neurotrophin 3, NTF3; and neurotrophin 4/5, NTF-4/5 (Ernfors et al. 1990, Lara et al. 1990, Berkemeier et al. 1991, Hallböök et al. 1991, Dissen et al. 1995,
1996), but also expresses the receptors for each of them (NGFR and the tyrosine kinase high-affinity receptors NTRK1, NTRK2, and NTRK3; Klein et al. 1989, Dissen et al. 1991, 1995, 1996, Lamballe et al. 1991, Paredes et al. 2004, Abir et al. 2005). More recent studies have made clear that the NTs and their respective receptors are expressed in feto-neonatal rodent ovaries and fetal human ovaries before the initiation of follicular assembly (Dissen et al. 1995, Anesetti et al. 2001, Anderson et al. 2002, Spears et al. 2003, Abir et al. 2005).

The importance of NGF in early follicular development was made evident by the reduction in the number of primary and secondary follicles, and the decreased expression of FSH receptor (FSHR) found at the end of the first postnatal week of life in Ngf-null mice (Dissen et al. 2001). These findings suggested that NGF not only promotes the early stages of follicle development, but also induces the initial biochemical differentiation of secondary follicles into gonadotropin-responsive structures. 
Thus far, a role for NTF3 and its high-affinity receptor NTRK3 in follicle formation or follicle development has not been revealed (Spears et al. 2003). However, using Ntrk2-null mice, we (Paredes et al. 2004) and others (Spears et al. 2003) have recently demonstrated that NTRK2 signaling is required for oocyte survival and preantral follicular development. Spears et al. (2003) employed mutant mice lacking the intracellular domain of the receptor and found that these animals have a significantly lower number of oocytes and primordial follicles than wild-type (WT) controls, suggesting that NTRK2 signaling is required for germ cell survival before initiation of follicular assembly. Employing mutant mice lacking all NTRK2 isoforms, we found that the ovaries of these mice (or those lacking both NTF-4/5 and BDNF) suffer a stage-selective deficiency in early follicular development that compromises the ability of follicles to grow beyond the primary stage. Proliferation of granulosa cells - required for this transition - and expression of FSHRs, which reflects the degree of biochemical differentiation of growing follicles, are reduced in these 'complete' Ntrk2-null mice. To determine the importance of NTRK2 receptors for subsequent follicular development, and because Ntrk2-null mice die within the first 2 weeks of postnatal life, we grafted the ovaries from 4- to 5-day-old $\mathrm{KO}$ animals under the kidney capsule of WT adult female mice and examined the ovaries 2 weeks later. To our surprise, the Ntrk2-null ovaries failed to sustain follicular development and showed a striking loss of follicular organization, preceded by massive oocyte death. These results indicate that NTRK2 receptors facilitate the early development of ovarian follicles, and that the receptors become critical for oocyte survival after follicular assembly.

While the importance of NTRK2 receptors in early ovarian development appears now unquestionable, the general consistency - but also the specific differences emerging from the aforementioned results (Spears et al. 2003, Paredes et al. 2004) raise an entirely new set of questions. For instance, the size of the primordial follicle population is relatively normal by the end of the first postnatal week of life in both Ntrk1- and Ntrk2-null mice (Dissen et al. 2001, Paredes et al. 2004), but it is significantly reduced in perinatal NTRK2-deficient ovaries (Spears et al. 2003), suggesting that NTRK2 and/or NTRK1 receptors may be required for the timely initiation of folliculogenesis. If this is the case, are NTRK2 and/or NTRK1 receptors required for oocyte survival before follicular formation? Are NTRK1 receptors, in addition to NTRK2 receptors, required for the acquisition of functional FSHRs? The present study addresses these questions and provides evidence that both NTRK1 and NTRK2 receptors contribute not only to the assembly of primordial follicles, but also to the subsequent development of the newly formed follicles, and to the acquisition of FSHRs by the growing follicles. In addition, these studies show that oocyte death does not increase before the initiation of folliculogenesis in the absence of either NTRK1 or NTRK2 receptors, and that ligand-mediated activation of NTRK2 receptors enhances the capacity of the infantile ovary to respond to FSH with synthesis of cyclin D2, a cell cycle protein underlying the proliferative effects of $\mathrm{FSH}$ on granulosa cells (Sicinski et al. 1996).

\section{Results}

\section{Absence of NT signaling delays follicle assembly}

It was previously shown that 7-day-old ovaries from $\mathrm{Ngf}^{-1-}$ mice and from mice lacking all isoforms of the NTRK2 receptor have a deficiency of early follicle development, but a relatively normal complement of primordial follicles (Romero et al. 2002, Paredes et al. 2004). To determine whether this apparent normalcy represents a true lack of an NT role in facilitating follicular assembly or, instead, reflects a delay of follicular formation, we examined the ovaries of 2-dayold mice carrying null mutations of the genes encoding NGF and its high-affinity NTRK1 receptor, in addition to mice lacking all NTRK2 receptor isoforms. In all cases, and despite of the strain-related differences in the absolute number of follicles present at this early age, the number of primordial follicles was significantly lower in the mutant ovaries than that in the ovaries of their respective WT littermates (Fig. 1A-C). In each case, a clear immaturity of the ovary was apparent, with differences in the size of the primordial follicle population more clearly manifested in ovaries lacking NGF or its NTRK1 receptor (Fig. 1D, G and E, H respectively) than in Ntrk2-deficient ovaries (Fig. 1F and I). The $\mathrm{KO}$ ovaries not only have a reduced number of primordial follicles (examples denoted by red arrows), but also appear to exhibit a greater number of nonencapsulated oocytes than WT ovaries (Fig. 1G, H and I versus $\mathrm{D}, \mathrm{E}$ and $\mathrm{F}$ ).

\section{The neonatal reduction in primordial follicles of Ntrk1 - and Ntrk2-null ovaries is not due to perinatal death of oocytes}

Because the absence of NTRK1 and NTRK2-mediated signaling may result in oocyte death similar to that reported in mutant mice lacking the intracellular domain of the NTRK2 receptor (Spears et al. 2003), we used a TUNEL assay to examine the ovaries of Ntrk $1^{-/-}$and $N$ trk2 $2^{-/}$mice on the day of birth, i.e. preceding the initiation of folliculogenesis, for signs of apoptosis. Very few, if any, apoptotic cells (green color) were detected (Fig. 2A-D, arrows), including apoptotic oocytes, which were rarely observed (Fig. 2A-D; higher magnification images in $\mathrm{E}-\mathrm{G})$. Neither Ntrk1 ${ }^{-/-}$nor $\mathrm{Ntrk} 2^{-/}$ovaries had a higher incidence of apoptotic oocytes than WT controls (Fig. 2A-D). This scarcity of somatic and germ 

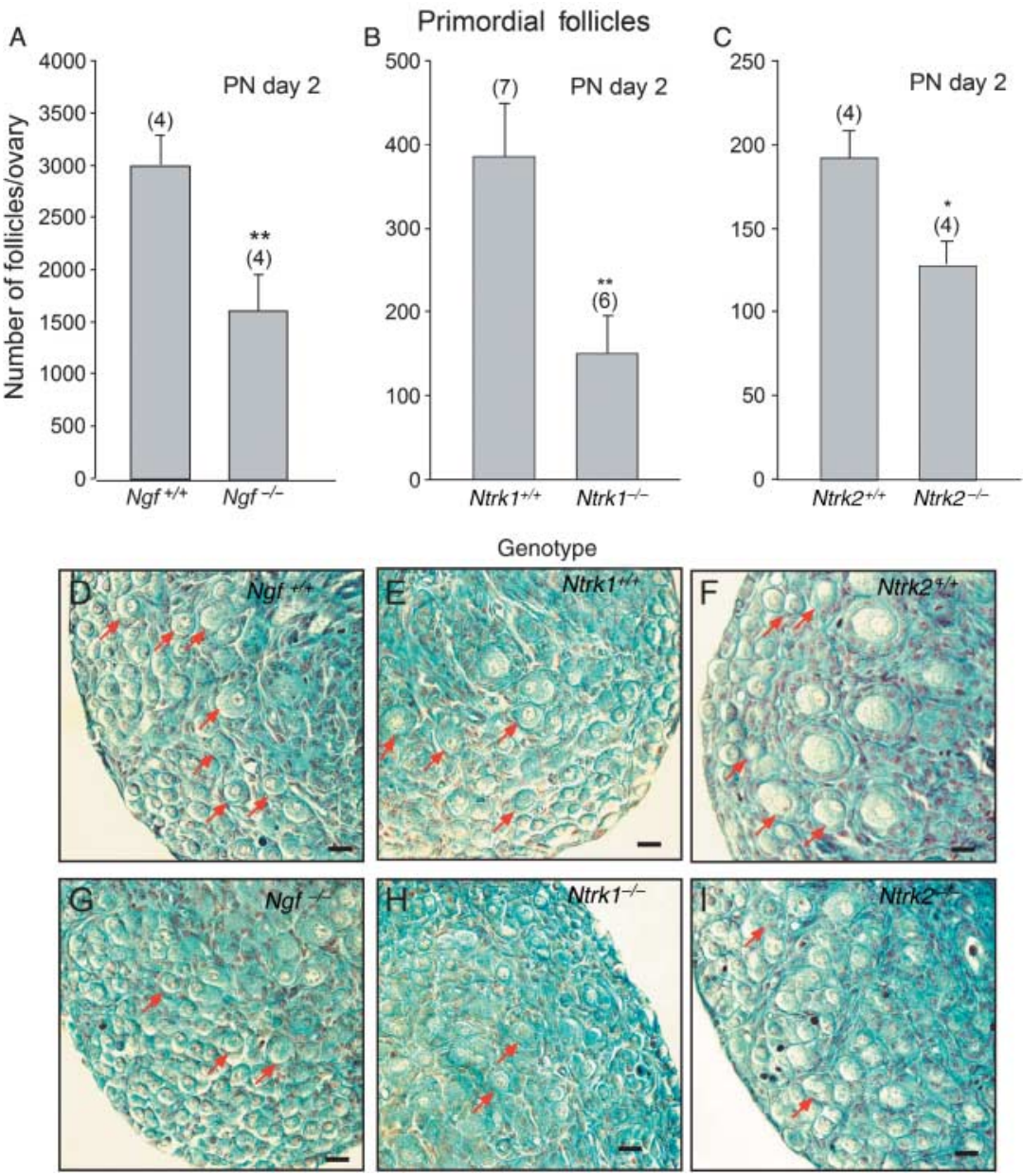

Figure 1 Formation of primordial follicles is reduced in the ovaries of mice lacking the Ngf, Ntrk1, or the Ntrk2 genes, as assessed by morphometric

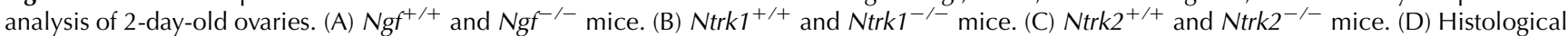
aspect of an $\mathrm{Ngf}^{+/+}$ovary showing an abundance of primordial follicles (examples denoted by red arrows), and the presence of primary follicles. (E) Similar aspect of an Ntrk $1^{+/+}$ovary. (F) An Ntrk2 $2^{+/+}$ovary showing that follicular development is more advanced in this strain at this time of postnatal life, with several primary follicles being apparent. (G) An Ngf ${ }^{-/-}$ovary showing a paucity of primordial follicles and many 'naked' oocytes. (H) An Ntrk1 ${ }^{-/-}$ovary also showing a deficiency of primordial follicles, and an accumulation of naked oocytes. (I) Similar aspect of an Ntrk $2^{-/-}$ovary. The numbers in parentheses above bars are number of animals per group, and vertical lines are S.E.M. ${ }^{*} P<0.05$ and $* * P<0.02$ versus WT control groups. Scale bars $=20 \mu \mathrm{m}$.

cell death is in contrast to 28-day-old ovaries (used as a positive control) in which granulosa cell apoptosis of an atretic follicle is widespread (Fig. $2 \mathrm{H}$ ). As expected, all cells were TUNEL positive in sections treated with DNAse I before performing the TUNEL reaction (Fig. 2I).

\section{NGF acting via NTRK1 receptors restores formation of primordial follicles in $\mathrm{Ngf}^{-1-}$ mice}

To determine whether the reduced number of primordial follicles observed in $\mathrm{Ngf}^{-1-}$ ovaries is specifically related to the absence of NGF-initiated signaling, we cultured ovaries from 1-day-old $\mathrm{Ngf}^{-/-}$mice for 4 days in the presence of NGF and determined the number of primordial follicles at the end of this period. Exposure to the NT distinctly $(P<0.01)$ increased the number of primordial follicles in comparison with untreated
$\mathrm{Ngf}^{-1-}$ ovaries (Fig. 3A). By contrast, NGF was ineffective in $\mathrm{Ntrk} 1^{-1}$ ovaries (Fig. 3B), indicating that the facilitatory effect of NGF on follicular assembly is mediated by NTRK1 receptors.

\section{Early follicle development is impaired in the absence of NTRK1 and NTRK2 receptors}

To determine whether the lack of NTRK1 and NTRK2 receptors begins to impact follicle development as the follicles enter the proliferative pool, we examined the ovaries of 4-day-old mutant mice and compared the number of primordial, primary, and secondary follicles with those of WT littermates. In both Ntrk1and Ntrk2-null ovaries, the size of the primordial follicle population was still significantly reduced $(P<0.02)$, but no significant differences in the number of primary 

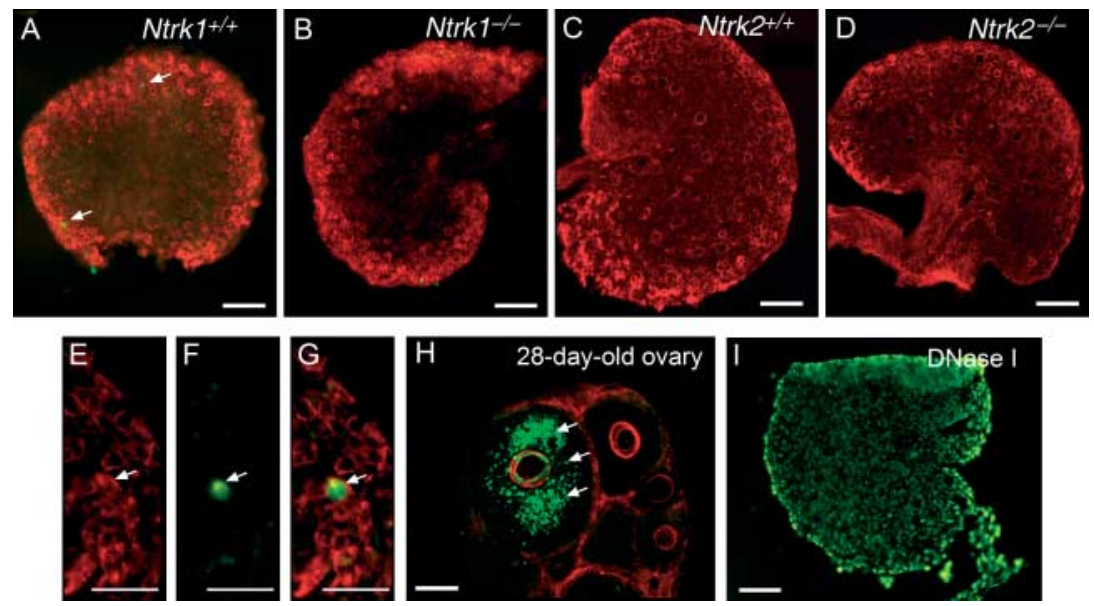

Figure 2 Representative sections from 0-day-old ovaries (day of birth) showing that the decreased number of primordial follicles observed in Ntrk1and Ntrk2-null ovaries is not due to an earlier, postnatal increase in apoptotic oocyte death. Apoptosis was detected using a TUNEL assay (green color) and oocytes were identified with a MAB against CD9, a membrane protein abundant in oocytes (red color). (A) Ntrk $1^{+/+}$ovary. (B) Ntrk $1^{-/-}$ ovary. (C) An Ntrk2 $2^{+/+}$ovary. (D) An Ntrk2 ${ }^{-1-}$ ovary. Arrows point to TUNEL-positive cells. (E) Higher magnification image showing a region of a WT ovary containing many naked oocytes. (F) Image showing that one of the oocytes shown in (E) is apoptotic (arrow). (G) Merged image. (H) Prepubertal 28-day-old ovary showing an abundance of apoptotic cell nuclei (green color, arrows) in granulosa cells of an atretic follicle, and the lack of apoptotic cells in healthy, adjacent follicles. (I) Section form a day-0 WT ovary treated with DNase I. Scale bars $=100 \mu \mathrm{m}$.

follicles were detected (Fig. 4A, B, D and E). By contrast, a marked decrease $(P<0.01)$ in the number of secondary follicles was observed in both mutants (Fig. 4C and F), suggesting that recruitment of primary follicles into the proliferative pool is compromised in the absence of either NTRK1 or NTRK2 signaling.

\section{Both NTRK1 and NTRK2 receptors contribute to the acquisition of FSHRs}

We previously showed that Fshr mRNA is decreased in the ovaries of $\mathrm{Ngf}^{-1-}$ and $\mathrm{Ntrk} 2^{-/-}$mice (Romero et al. 2002, Paredes et al. 2004), and that a short-term (8 h) exposure to NGF suffices to induce Fshr gene expression (Paredes et al. 2004). To determine whether the supportive effect of NGF on Fshr gene expression is mediated by high-affinity NTRK1 receptors or by the common NT receptor NGFR, we compared the abundance of Fshr mRNA in the ovaries of 7-day-old Ntrk $1^{-1-}$ and $\mathrm{Ngfr}^{-1-}$ mice to that of WT littermates. As shown in Fig. 5, the absence of NTRK1 (A), but not that of NGFR (B) resulted in significantly $(P<0.05)$ lower Fshr mRNA levels than in WT ovaries, suggesting that NGF maintains FSHR expression (Romero et al. 2002) via activation of NTRK1 receptors.

By the end of the first week of life, the ovaries from $\mathrm{Ntrk} 2^{-1-}$ mice show a marked reduction in granulosa cell proliferation, and a sustained reduction in the number of secondary follicles (Paredes et al. 2004). It was, therefore, important to determine: a) whether a transient exposure to the NTRK2 ligand NTF4 can increase Fshr gene expression, as previously shown for NGF (Romero et al. 2002), and b) if this treatment also results in the formation of FSHRs able to initiate a proliferative signal, as measured by the ability of FSH to increase the synthesis of cyclin D2, a cell cycle protein known to mediate the stimulatory effect of FSH on granulosa cell proliferation (Sicinski et al. 1996). A short (8-h) in vitro exposure of 4-day-old WT ovaries to NTF4 $(100 \mathrm{ng} / \mathrm{ml})$ significantly $(P<0.01)$ increased $F$ shr mRNA levels (Fig. 6A). Ovaries treated with NTF4 for $8 \mathrm{~h}$ and then with $\mathrm{FSH}$ for $24 \mathrm{~h}$, in the absence of the NT, responded to $\mathrm{FSH}$ with an increase $(P<0.05)$ in cyclin D2 levels (Fig. 6B). This increase was not seen in ovaries treated with NTF4 for $8 \mathrm{~h}$ and then cultured in medium

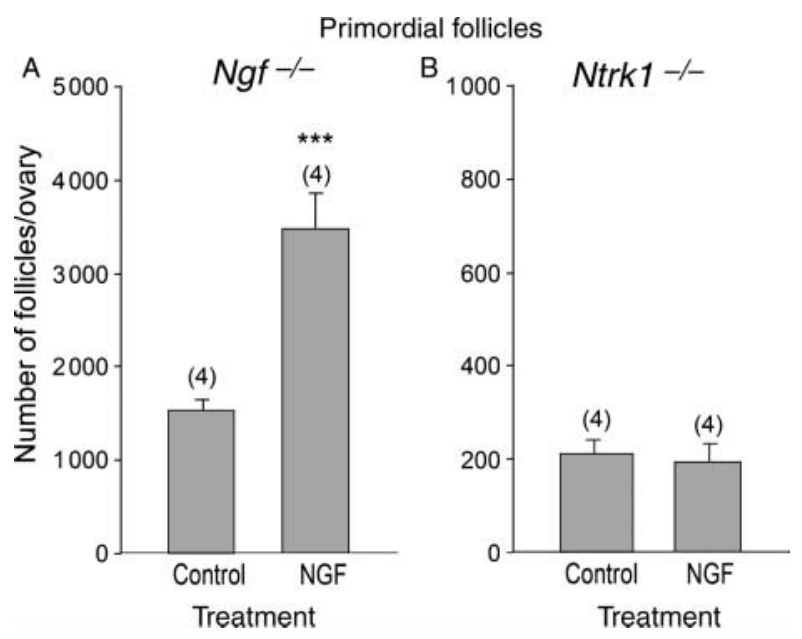

Figure 3 NGF acting via NTRK1 receptors rescues the number of primordial follicles that fail to form in $\mathrm{Ngf}^{-/}$mice. (A) The number of primordial follicles is increased in $\mathrm{Ngf}^{-1-}$ ovaries explanted on the day of birth (day 0) after a 4-day exposure to NGF (100 ng/ml). (B) The same treatment is ineffective in the absence of NTRK1 receptors. Vertical lines represent the S.E.M. and the numbers in parentheses are number of animals per group. ${ }^{* * *} P<0.01$ versus untreated control group. 

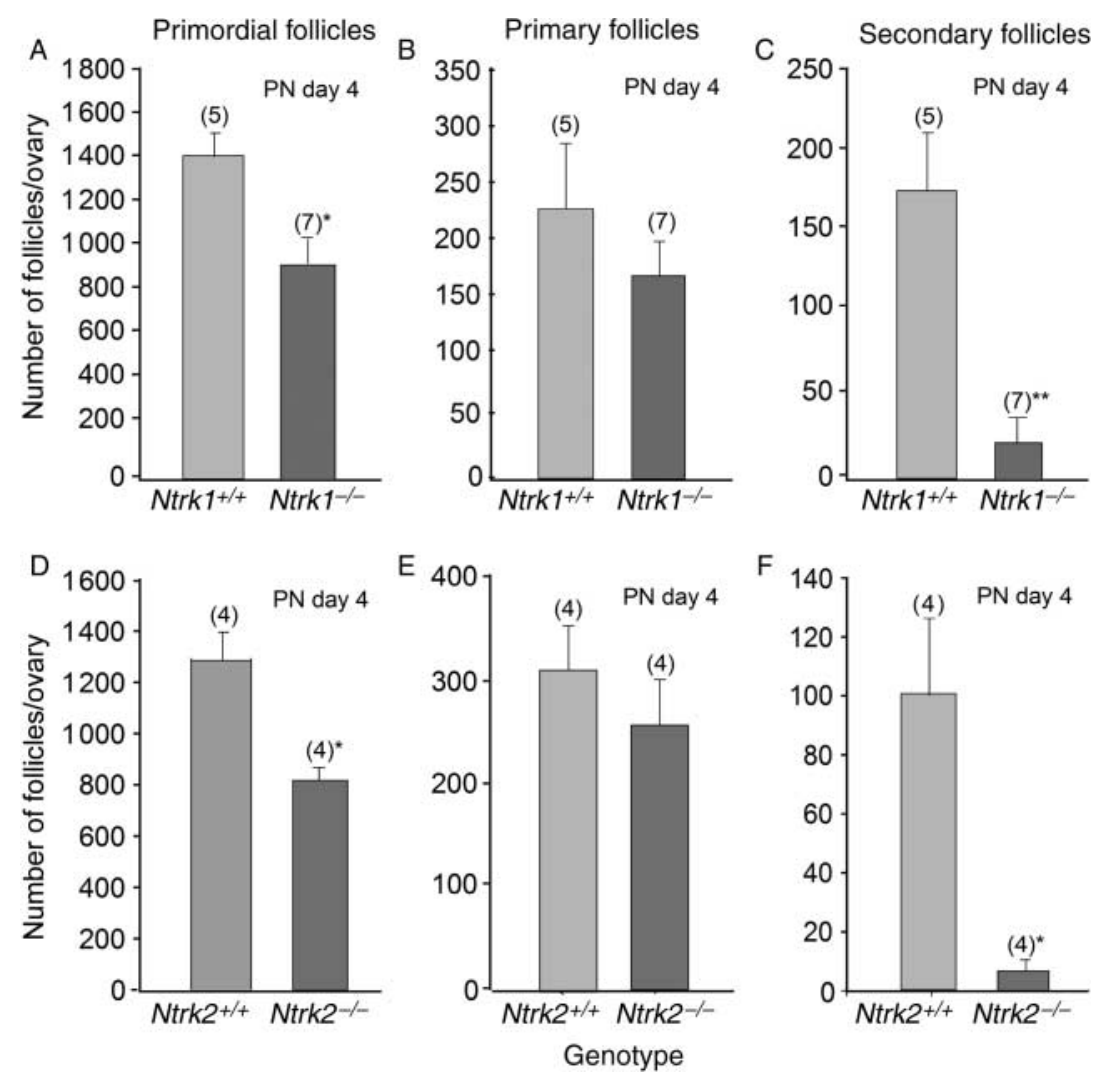

Figure $4 \mathrm{~A}$ decreased number of primordial follicles persist 4 days after birth in both $N \operatorname{trk} 1^{-/-}$and Ntrk2 $2^{-/-}$ovaries, which in addition show a reduced number of secondary follicles. (A-C) Ntrk1 $1^{+/+}$and $N t r k 1^{-/-}$ovaries. (D-F) Ntrk2 ${ }^{+/+}$and Ntrk2 ${ }^{-/-}$ovaries. Vertical lines represent S.E.M. and the numbers in parentheses on top of bars are number of animals per group. ${ }^{*} P<0.05$ and ${ }^{* *} P<0.02$ versus respective WT groups.

alone for $24 \mathrm{~h}$ or in ovaries cultured in medium alone for $8 \mathrm{~h}$ and then with FSH for $24 \mathrm{~h}$ (Fig. 6B). These results suggest that the supportive effect of NTRK2-mediated signaling on follicle development is mediated, at least in part, by facilitating the formation of biologically active FSHRs capable of increasing the expression of a key cell cycle protein underlying the proliferative effect of FSH on the developing ovary.

\section{Discussion}

The present results demonstrate that mouse ovaries lacking either NTRK1 or NTRK2 receptors exhibit a reduced number of primordial follicles and a deficiency in early follicular development. The results also indicate that the defect in primordial follicle assembly is not due to premature oocyte death. Instead, it appears to results from an NT deficiency-dependent inability of somatic and germ cells to engage into the bidirectional communication process required for follicular organization. The subsequent delay of follicle development is likely related to the absence of an NT-dependent proliferative signal followed by reduced formation of FSHR.

The observation that folliculogenesis is impaired in mice lacking either NGF or its NTRK1 receptor complements and expands the results of a previous study examining follicular development in $\mathrm{Ngf}^{-1-}$ mice (Dissen et al. 2001). In that study, we found that the number of primordial follicles of $\mathrm{Ngf} \mathrm{KO}$ ovaries collected on postnatal day 7 was similar to that of WT littermates, implying that follicular assembly does not require NGF. However, an increased number of 'naked' (i.e. non-encapsulated) oocytes were also noted, suggesting that, instead of preventing follicle assembly, the absence of NGF-mediated signaling may have delayed primordial follicle formation. The present results, obtained using ovaries collected at the time of initiation of folliculogenesis (PN day 2) and when follicle assembly is progressing in earnest (PN day 4), support this notion. Our findings also indicate that the supportive effect of NGF on follicular formation is mediated by NTRK1 receptors, because the absence of these receptors results in an ovarian phenotype indistinguishable from that of $\mathrm{Ngf}^{-1-}$ mice.

Confirming the findings of Spears et al. (2003), we have now observed that the ovaries of 2- and 4-day-old Ntrk $2^{-1-}$ mice contain fewer primordial follicles that WT controls. When the ovaries were examined at a later age (PN day 7), only a trend toward a lower number of primordial follicles was observed (Paredes et al. 2004). Together, these earlier findings and the present observations indicate that - as is the case of NGF- and 


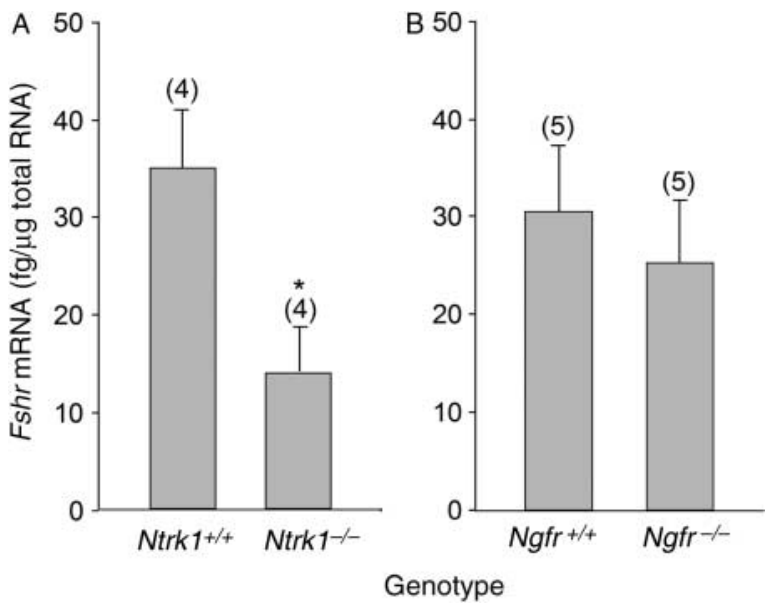

Figure 5 Fshr mRNA abundance is sustained by NTRK1-mediated signaling, but does not require NGFR. (A) Fshr mRNA levels in 7-day-old Ntrk $1^{-1-}$ ovaries and their age-matched $\mathrm{Ntrk} 1^{+/+}$ controls. (B) Fshr mRNA in Ngfr ${ }^{-1-}$ ovaries and their age-matched WT controls. Vertical lines represent S.E.M. and the numbers in parentheses on top of bars are number of animals per group. ${ }^{*} P<0.05$ versus Ntrk $1^{+/+}$group.

NTRK1-deficient mice - the absence of NTRK2mediated signaling delays, but does not prevent, follicle assembly.

Mice lacking the intracellular domain of the NTRK2 receptor show a reduced number of oocytes 4-6 days after birth (Spears et al. 2003), suggesting that the lower number of primordial follicles observed in these animals may be a consequence of prior oocyte death, which would reduce the pool of oocytes able to organize somatic cells into primordial follicles. We have now examined the ovaries of $\mathrm{Ntrk} 1^{-/-}$and $\mathrm{Ntrk} 2^{-/-}$mice lacking all isoforms of the NTRK2 receptor on the day of birth, i.e. $48 \mathrm{~h}$ before the initiation of folliculogenesis, and found no evidence for an increased incidence of oocyte death. In fact, we detected very few apoptotic oocytes in WT ovaries, and this low number remained unchanged in both $\mathrm{Ntrk} 1^{-/-}$and $\mathrm{Ntrk} 2^{-/-}$ovaries. Moreover, using $\mathrm{Ngf}^{-1-}$ mice as a model to define the effects of NTs on primordial follicle formation, we observed that ovaries treated with NGF from the day of birth respond to the NT with increased formation of primordial follicles, but only when NTRK 1 receptors are present. These findings indicate that the loss of primordial follicles resulting from the absence of NT signaling can be prevented by restoring NTs to the deficient ovary, as long as the corresponding high-affinity NTRK receptors are also present. This conclusion does not exclude the possibility of a prenatal increase in oocyte death caused by the absence of NTRK receptors. Such a loss, if it occurs, may exacerbate the natural loss of oocytes that occurs before birth (McClellan et al. 2003). Because in the present study we did not count the total number of 'non-encapsulated' follicles present on the day of birth, we cannot formally rule out this possibility. Nevertheless, our findings are consistent with the notion that the loss of primordial follicles seen in NT/ NTRK receptor-deficient ovaries is not an irretrievable event, because it can be reversed by exposing the ovary to an NT (NGF), and it is no longer evident by the end of the first postnatal week of life (Dissen et al. 2001, Paredes et al. 2004). The cellular mechanisms underlying the supportive effect of NTs on primordial follicle formation remain to be identified.

Both Ntrk1- and Ntrk2-null ovaries, in addition to the ovaries from $\mathrm{Ngf}^{-1-}$ mice, exhibit a reduced number of secondary follicles. Although 7-day-old $\mathrm{Ngf}^{-1-}$ ovaries also have a reduced number of primary follicles (Dissen et al. 2001), this was not the case of the younger ovaries examined in the present study. The most parsimonious
A

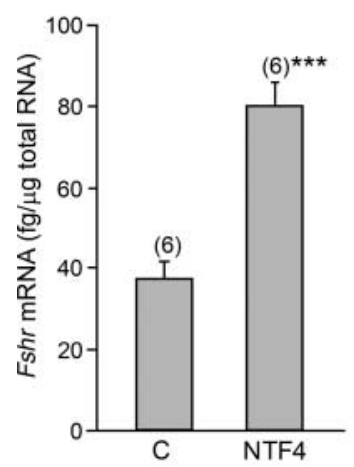

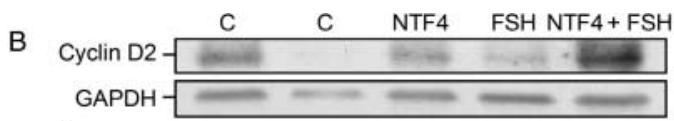

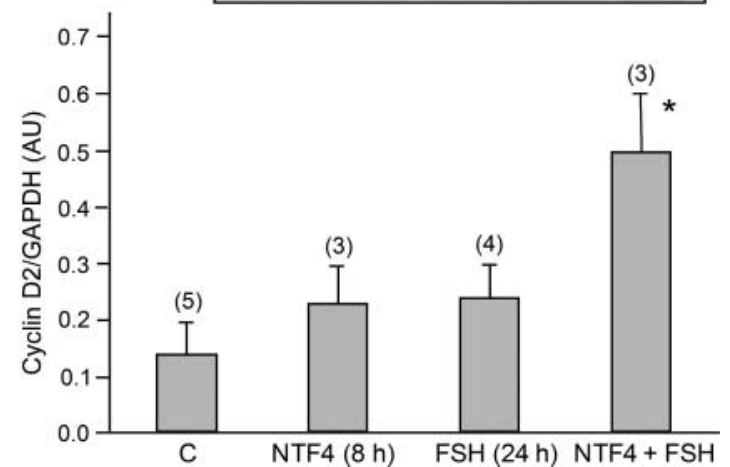

Figure 6 NTF4 increases Fshr mRNA levels and induces the formation of biologically active FSHR (as measured by the ability of FSH to increase the synthesis of cyclin D2, an FSH-responsive gene) in explanted ovaries from 4-day-old mice. (A) FshrmRNA abundance, determined by real-time PCR, increases after an 8-h exposure to NTF4 (100 ng/ml). (B) A short-term (8-h) exposure to NTF4 (100 ng/ml) enhances the ability of the ovary to respond to $\mathrm{FSH}(500 \mathrm{ng} / \mathrm{ml})$ with an increase in cyclin D2 formation, as determined by western blot analysis. A representative western blot is shown on top of the bar graph depicting the quantitative analysis of this experiment. Vertical lines are S.E.M. and the numbers in parentheses above bars are number of animals per group. ${ }^{*} P<0.05 ;{ }^{* *} P<0.01$ versus untreated control groups (C). 
explanation for this difference is that the size of the primary follicle population reflects an equilibrium between the number of primordial follicles that differentiate into follicles containing one-cell layer of cuboidal granulosa cells and those that enter the proliferative pool. If a defect in primordial-to-primary follicle differentiation is more pronounced than a defect in primary follicle recruitment to the proliferative pool (primary to secondary follicle transition), as it happens in 7-day-old NGF-deficient mice (Dissen et al. 2001), the number of primary follicles is reduced. However, if the recruitment defect is more prevalent, then the number of primary follicles may not change.

The cellular mechanisms underlying the supportive effect of NTs on follicle development have not been elucidated. However, in both NGF- and NTRK2deficient ovaries, somatic cells proliferation is reduced (Romero et al. 2002, Paredes et al. 2004), suggesting that follicle development is stunted because follicular cells fail to proliferate. This functional relationship appears more obvious in the case of the NTRK2 receptor, because the proliferative defect observed in NTRK2deficient ovaries takes place in the granulosa cell compartment (Paredes et al. 2004). The present results show that a transient ( $8 \mathrm{~h}$ ) exposure of 4-day-old ovaries to NTF4 enhances the ability of the ovary to respond to FSH with formation of cyclin D2, a cell cycle protein known to mediate the stimulatory effect of FSH on granulosa cell proliferation (Sicinski et al. 1996). Such an effect of NTF4, coupled to the proliferative deficit observed in Ntrk2-null ovaries, strongly suggest that one mechanism by which activation of NTRK2 receptors supports follicular development is by endowing the developing follicles with the capacity to respond to FSH with proliferation. It is also clear, however, that the defect in follicle development becomes evident before the follicles reach the secondary stage and, consequently, acquire responsiveness to FSH (Dierich et al. 1998). This early deficiency strongly suggests that NTs exert proliferative actions on their own, in the absence of $\mathrm{FSH}$. The present report does not directly address this likely possibility, which needs to be examined in detail by future studies.

In contrast to $\mathrm{Ntrk} 2^{-1-}$ ovaries, the ovaries of $\mathrm{Ngf}{ }^{-1-}$ mice show a reduced proliferation of mesenchymal cells (Romero et al. 2002), implying the existence of a directional communication pathway that, initiated in these cells and acting on granulosa cell/oocytes, supports follicle development. Such a pathway was previously postulated based on morphological evidence (Rajah et al. 1992), but the molecules involved remain to be identified.

In mice lacking FSHR, follicular development proceeds normally until follicles reach the secondary stage (Dierich et al. 1998), indicating that they become gonadotropin dependent only at this time. Our results suggest that NTs acting via NTRK receptors are intraovarian factors that promote this biochemical differentiation process because FSHR expression is low in the absence of NGF (Romero et al. 2002) or its NTRK1 receptor (present results), and also in ovaries lacking NTRK2 receptors (Paredes et al. 2004). Conversely, Fshr mRNA levels increase within $8 \mathrm{~h}$ of exposing ovaries to either NGF, the NTRK1 ligand (Romero et al. 2002) or NTF4 (present results), one of the NTRK2 ligands. The common NT receptor NGFR does not appear to play a role in this process, because Ngfr-null mice have normal Fshr mRNA levels. This result was not entirely unexpected because in the rodent ovary NGFR is expressed only in mesenchymal cells long before the initiation of follicle assembly (Dissen et al. 1995), and remains highly expressed in thecal cells throughout the natural history of follicle development (Dissen et al. 1991). Because the loss of NGFR expression is only partial in existing Ngfr KOs (von Schack et al. 2001, Paul et al. 2004), it may be necessary to use conditional KO mice in which the cell-specific loss of the receptor is complete to fully understand the contribution that this receptor may have to ovarian development.

The cell-to-cell signaling pathways underlying the action of NTs on follicle organization and subsequent development are not known. Because in the perinatal mouse ovary NTRK receptors are located in both somatic and germ cells (Dissen et al. 2001, Anderson et al. 2002, Paredes et al. 2004, Abir et al. 2005), it appears plausible that part of the mechanism used by NTs to facilitate folliculogenesis and the progression of follicular development to the secondary stage involves the activation of reciprocal mesenchyma-granulosa cell and granulosa cell-oocyte communication pathways. The nature of the cell-cell signaling molecules involved remains to be established, but in recent studies examining the changes in gene expression that accompany the absence of NTRK1 and NTRK2 receptors, we have observed that a cell-cell communication pathway affected in both mutant ovaries involves the Jagged1 ligand and its Notch receptor (Kerr et al. 2006).

Altogether, the present results provided substantial support to the concept that NTs are intraovarian factors that promote ovarian development before the ovary becomes subjected to gonadotropin control.

\section{Materials and Methods}

\section{Ntrk1 -, Ntrk2-, Ngfr-, and Ngf-null mice}

Ntrk1 ${ }^{+/-}(129 S v-C 57 B L / 6)$ mice (Liebl et al. 2000) were a generous gift of Dr Lino Tessarollo (National Cancer Institute, Frederick, MD, USA). Ngf ${ }^{+/-}(\mathrm{C} 57 \mathrm{BL} / 6-\mathrm{AB} 1)$ mice (Crowley et al. 1994) were kindly provided by Dr Heike Phillips (University of California at San Francisco, CA, USA) and $\mathrm{Ngfr}^{+/-}$(BALB/C) mice (Lee et al. 1992) were generously provided by Dr Kuo-Fen Lee (The Salk Institute, La Jolla, 
CA, USA). Ntrk2 ${ }^{+/-}$mice (C57BL6-DBA) were generated as previously described (Paredes et al. 2004). The mutant mice were bred to WT animals of the same genetic background, and the null mutant and WT mice used in this study were obtained by crossing F1 heterozygous individuals. The animals were housed under controlled conditions of temperature $\left(23-25{ }^{\circ} \mathrm{C}\right)$ and light (12 h light:12 h darkness; lights on from 0700 to $1900 \mathrm{~h}$ ), and were given ad libitum access to food (LabDiet 5001, PMI Nutrition International Brentwood, St Louis, MO, USA) and tap water. The use of mice was duly approved by the ONPRC Animal Care and Use Committee, in accordance to the guidelines provided by the NIH Guide for the Care and Use of Laboratory Animals.

\section{Collection of ovarian tissue and genotyping}

Ovaries from entire litters were collected at 0 (day of birth), 2 and 4 days after birth, and assigned to different procedures (organ culture, RNA extraction or morphometric analysis) before establishing each genotype. Once this was known, the ovaries were assigned to either a WT or a KO group. No heterozygotes were studied. Genotyping was performed via PCR analysis of tail DNA, employing specifics oligodeoxynucleotide primers that amplify a DNA segment comprising both the targeting vector and the gene sequence specific to each mutant animal (Lee et al. 1992, Crowley et al. 1994, Liebl et al. 2000, Paredes et al. 2004).

\section{Culture of ovaries}

To determine the effect of NGF on primordial follicle formation, ovaries from 0 -day-old $\mathrm{Ngf}^{-/-}$and $\mathrm{Ntrk} 1^{-1-}$ mice were dissected under a stereomicroscope using aseptic conditions, placed on sterile lens paper and cultured on metal grids in a 24-well plate at the interface of air/culture medium, under an atmosphere of $60 \% \quad \mathrm{O}_{2}-35 \% \quad \mathrm{~N}_{2}-5 \% \quad \mathrm{CO}_{2}$, as described (Romero et al. 2002, Paredes et al. 2004). One ovary from each animal was cultured in presence of NGF $(100 \mathrm{ng} / \mathrm{ml})$ for 4 days; the contralateral ovary served as an untreated control. At the end of this period, the ovaries were collected, fixed in Kahle's fixative (Hirshfield \& DeSanti 1995), embedded in paraffin, serially sectioned at $6 \mu \mathrm{m}$ and stained for morphometric analysis, as reported earlier (Dissen et al. 2001).

To determine the effect of NTRK2 activation on Fshr mRNA levels, and on cyclin D2 abundance, ovaries from 4-day-old WT mice were dissected and cultured as outlined above. When used for measurement of Fshr mRNA, the ovaries were cultured for $8 \mathrm{~h}$ in presence or absence of NTF4 (100 ng/ml), then frozen in dry ice, and stored at $-85^{\circ} \mathrm{C}$ until RNA extraction. Cultures employed for western blot analysis of cyclin D2 protein levels were subjected to the same experimental protocol we used earlier to document an effect of NGF on the formation of functional FSHR (Romero et al. 2002). In brief, the ovaries were treated with NTF4 for $8 \mathrm{~h}$ or left untreated. At the end of this period, the medium was replaced with fresh medium alone or medium containing FSH (500 ng/ml), in the absence of NTF4. Twenty-four h later, the ovaries were collected, frozen on dry ice, and stored at $-85^{\circ} \mathrm{C}$ until protein extraction.

\section{RNA extraction and real-time PCR}

Total RNA was isolated from cultured ovaries treated with NTF4 using Tri-Reagent (Molecular Research Center, Inc., Cincinnati, $\mathrm{OH}$, USA) as previously described (Paredes et al. 2004). Fshr mRNA was detected by real-time PCR using a procedure previously described (Romero et al. 2002) with some modifications. After reverse transcribing 200 ng of total RNA, aliquots of each reaction (10 ng $\mathrm{cDNA} / \mu \mathrm{l}$ ) were diluted 1:10 before using $2 \mu \mathrm{l}$ for real-time PCR. Each sample was run in triplicate along with a relative and an absolute standard curve. Relative standard curves, generated by serially diluting one sample 1:10 to 1:10 000 times, served to estimate the content of 18S rRNA of each sample. The primers used to detect 18s rRNA were purchased as a kit (TaqMan Ribosomal RNA Control Reagents Kit, Perkin Elmer Applied Biosystems, Foster City, CA, USA). Absolute standard curves were constructed by using serial dilutions (1:10) of sense Fshr RNA (2 ag-2 ng, see below). The threshold cycle number $\left(C_{\mathrm{t}}\right)$ from each sample was referred to this curve to estimate the corresponding RNA content, and each RNA value was then normalized for procedural losses using the $18 \mathrm{~S}$ rRNA values estimated from the relative standard curve. $C_{\mathrm{t}}$ was the fractional cycle number at which the fluorescence accumulated to a level 10 times $>1$ s.D. from basal values. The Fshr and 18s rRNA primers employed were those already reported (Romero et al. 2002). These primers and the fluorescent probes for real-time PCR were selected with the assistance of the program, Primer Express (Perkin Elmer Applied Biosystems). Real-time PCRs were performed in a total volume of $10 \mu \mathrm{l}$, each reaction containing $2 \mu$ of the diluted reverse transcribed sample or $2 \mu \mathrm{l}$ of sense Fshr mRNA standard, $5 \mu \mathrm{l}$ TaqMan Universal PCR Master Mix (Perkin Elmer Applied Biosystems), $250 \mathrm{nM}$ of each gene specific- and ribosomal fluorescent probes, $300 \mathrm{nM}$ of each gene specific primer, and $10 \mathrm{nM}$ of each ribosomal primer.

To construct the sense Fshr RNA standard curve used to quantify Fshr mRNA abundance in the ovary samples, we used a 346 bp cDNA generated by RT-PCR of total RNA derived from adult mouse ovaries. The primers used were a $5^{\prime}$ forward primer (5'-GCCCTGGCCTTTGTGGTCATCTGT-3') corresponding to nucleotides 1675-1698 in mouse Fshr mRNA (NM_013523) and a $3^{\prime}$ reverse primer (5'-AAATCTGGGCTTGCACCTCATAAC-3') complementary to nucleotides 1997-2020. To amplify this fragment, we used a PCR amplification protocol consisting of 33 cycles of denaturing at $94{ }^{\circ} \mathrm{C}(30 \mathrm{~s})$, annealing at $57{ }^{\circ} \mathrm{C}(60 \mathrm{~s})$, and extension at $72{ }^{\circ} \mathrm{C}(60 \mathrm{~s})$. The resulting PCR product was cloned into the pGEM-T vector (Promega Corp.), and sequenced from both ends to verify its identity.

\section{Morphometric analysis}

Ovaries from 2- and 4-day-old mice, and 0-day-old ovaries maintained for 4 days in organ culture were fixed in Kahle's fixative, embedded in paraffin, serially sectioned at $6 \mu \mathrm{m}$, stained with Weigert's iron hematoxylin, and counterstained with picric acid-methyl blue, as reported (Dissen et al. 2001, Romero et al. 2002, Paredes et al. 2004). Every other section was imaged as described (Paredes et al. 2004) and the degree of follicle development was morphometrically analyzed counting only follicles in which the nucleus of the oocyte was visible 
(Paredes et al. 2004). The total number of follicles per ovary was then calculated by first dividing the total number of follicles counted per ovary by the number of sections examined and then multiplying this number by the total number of sections made from each ovary. Because each ovary yielded about 140 sections, we counted about 50 sections per ovary. All counts were performed without previous knowledge of the animal's genotype. The follicles were classified in different developmental stages according to well-established criteria (Peters 1969) that we have previously used (Dissen et al. 2001, Romero et al. 2002, Paredes et al. 2004). Briefly, primordial follicles are the initial result of follicular assembly; they contain an oocyte surrounded by a single layer of flattened pregranulosa cells (Peters 1969). Primordial follicles become primary follicles (type 3a; Peters 1969) by a process that results in the differentiation of the flattened granulosa cells into a cuboidal morphology (Peters 1969, Hirshfield 1991). Granulosa cell proliferation and oocyte growth begin at this point resulting in the formation of larger (type $3 \mathrm{~b}$ ) primary follicles first, and secondary follicles with two (type 4) or more layers of granulosa cells (type 5 and larger) subsequently.

\section{Assessment of apoptosis}

To determine whether the decrease in the number of primordial follicles observed in Ntrk $1^{-1-}$ and Ntrk2 $2^{-1-}$ mice on postnatal days 2 and 4 is due to death of oocytes before they become organized into follicular structures, we used ovaries from 0-day-old mice. The ovaries were immersed in Zamboni's fixative overnight at $4{ }^{\circ} \mathrm{C}$ and processed as described (Paredes et al. 2004), before preparing $14 \mu \mathrm{m}$ cryostat sections. Six-eight randomly selected sections derived from three mice per group were then subjected to combined immunohistofluorescence-TUNEL. Oocytes were identified using a rat MAB (Mab KMC8, $5 \mu \mathrm{g} / \mathrm{ml}$, BD Pharmigen, San Diego, CA, USA) that recognizes mouse CD9, a member of the tetraspanin family of membrane proteins expressed in the plasma membrane of oocytes (Zhu et al. 2002). CD9 is also present in several cell types of the immune system (BD Pharmigen, product specifications). After an overnight incubation at $4{ }^{\circ} \mathrm{C}$ with the CD9 antibody, the immunoreaction was developed by incubating the sections for $1 \mathrm{~h}$ at room temperature with Alexa 594 donkey anti-rat gamma globulin (1:500). Following extensive rinsing, the sections were subjected to the TUNEL reaction, which was performed following the manufacturer's instructions. To detect apoptotic ovarian cells by TUNEL, we used an in situ cell death detection kit coupled with fluorescein detection (Roche Diagnostics Co). Positive controls for the TUNEL reaction included sections from the ovary of a 28-dayold mouse (to detect apoptotic granulosa cells) and sections from day- 0 ovaries treated with DNase I (Ambion, Austin, TX, USA; $10 \mathrm{U} / \mathrm{ml}$ for $20 \mathrm{~min}$ at room temperature).

\section{Western blots}

Proteins and RNA derived from the experiments described in the subsection 'Culture of ovaries' were extracted simultaneously, as recommended (Morse et al. 2006, Tolosa et al. 2007) using a nucleic acid column extraction kit
(RNeasy minikit, Qiagen Inc). Briefly, pools of four ovaries (i.e. from two animals) were homogenized using an ultraTurrax homogeneizer in $300 \mu \mathrm{l}$ of a guanidine hydrochloridecontaining buffer (RLT buffer), provided with the kit supplemented with $10 \% \quad \beta$-mercaptoethanol. The RNA was then extracted using the RNeasy minicolumn, following the manufacturer's instructions. The flow-through containing the proteins was collected at every step and pooled. The proteins were precipitated overnight at $-20{ }^{\circ} \mathrm{C}$, the precipitates were collected by centrifugation at $10000 \mathrm{~g}\left(15 \mathrm{~min}\right.$ at $\left.4{ }^{\circ} \mathrm{C}\right)$, and the pellets were washed three times with $100 \%$ cold ethanol (each wash for $30 \mathrm{~min}$ at $-20^{\circ} \mathrm{C}$ ), before collecting the proteins by centrifugation at $10000 \mathrm{~g}$ for $30 \mathrm{~min}$ at $4{ }^{\circ} \mathrm{C}$. Thereafter, the pellets were air-dried and each sample was resuspended in 40-50 $\mu$ Laemmli sample buffer (187 mM Tris-base, 9\% SDS, $15 \%$ glycerol, and $15 \% \beta$-mercaptoethanol, $\mathrm{pH} 6.8$ ), boiled $5 \mathrm{~min}$, and fractionated in a $4-20 \%$ precast SDS-PAGE gel (Invitrogen). Due to the small amount of tissue per sample, the protein concentration of each sample was not determined before gel loading. After electrophoresis at $130 \mathrm{~V}$ for $2 \mathrm{~h}$, the proteins were transferred for $1.5 \mathrm{~h}$ at $4{ }^{\circ} \mathrm{C}$ onto PVDF membranes (Millipore, Billerica, MA, USA). The membranes were blocked in 5\% non-fat milk for $1 \mathrm{~h}$. A rat MAB raised against recombinant cyclin D2 (34B1-3; Santa Cruz Biotechnology, Santa Cruz, CA, USA) was used at a 1:1000 dilution (overnight at $4{ }^{\circ} \mathrm{C}$ ) followed by a goat anti-rat HRP antibody (1 h at room temperature, 1:25 000 sc-2006, Santa Cruz Biotechnology). The signal was developed by ECL using the Super Signal West Dura Extended Duration Substrate (Pierce Biotechnology Inc., Thermo Fisher Scientific Inc., Rockford, IL, USA). For quantitation purposes, the membrane was washed several times in Tris-buffered saline $0.5 \%$ Tween20 before an overnight exposure (at $4{ }^{\circ} \mathrm{C}$ ) to a mouse $\mathrm{MAB}$ against GAPDH (Abcam Inc., Cambridge, MA, USA; 1:10 000 dilution), followed by an anti-mouse HRP antibody (Invitrogen; $1 \mathrm{~h}$ at room temperature, 1:50 000 dilution). To develop the signal, an ECL substrate (Western Lighting, Perkin Elmer) was used.

\section{Statistical analysis}

Before specific analyses were performed, the data were subjected to a normality test. Data passing this test were then analyzed using the two-tailed Student's $t$-test to assess the differences between two groups of animals or independent observations, or a one-way ANOVA followed by the StudentNeuman-Keuls multiple comparison test for unequal replications, when comparing several groups. When the data failed the normality test, they were analyzed using an ANOVA test on ranks followed by the Kruskal-Wallis one-way ANOVA on ranks test (SigmaStat, Systat Software Inc., v3.11, San Jose, CA, USA). A $P$ value $<0.05$ was considered statistically significant.

\section{Declaration of interest}

The authors declare that there is no conflict of interest that could be perceived as prejudicing the impartiality of the research reported. 


\section{Funding}

This work was supported by NIH grants HD24870 (SRO), the Eunice Kennedy Shriver NICHD/NIH through cooperative agreement HD18185 as part of the Specialized Cooperative Centers Program in Reproduction and Infertility Research (SRO), and RR000163-49 for the operation of the Oregon National Primate Research Center (GAD, SRO). CG-R was a visiting scientist and BK was a postdoctoral fellow, both supported by the NICHD TW/HD00668 Fogarty International Training \& Research in Population \& Health grant.

\section{Acknowledgements}

We thank Mr Ricardo A Ojeda for morphometric analysis of ovarian follicles.

\section{References}

Abir R, Fisch B, Jin G, Barnnet $M$, Ben-Haroush A, Felz C, Kessler-Icekson G, Feldberg D, Nitke S \& Ao A 2005 Presence of NGF and its receptors in ovaries from human fetuses and adults. Molecular Human Reproduction 11 229-236.

Anderson RA, Robnson LLL, Brooks J \& Spears N 2002 Neurotropins and their receptors are expressed in the human fetal ovary. Journal of Clinical Endocrinology and Metabolism 87 890-897.

Anesetti G, Lombide P, D'Albora H \& Ojeda SR 2001 Intrinsic neurons in the human ovary. Cell and Tissue Research 306 231-237.

Berkemeier LR, Winslow JW, Kaplan DR, Nikolics K, Goeddel DV \& Rosenthal A 1991 Neurotrophin-5: a novel neurotrophic factor that activates trk and trkB. Neuron 7 857-866.

Crowley C, Spencer SD, Nishimura MC, Chen KS, Pitts-Meek S, Armanini MP, Ling LH, McMahon SB, Shelton DL, Levinson AD et al. 1994 Mice lacking nerve growth factor display perinatal loss of sensory and sympathetic neurons yet develop basal forebrain cholinergic neurons. Cell 76 1001-1011.

Dierich A, Sairam MR, Monaco L, Fimia GM, Gansmuller A, LeMeur M \& Sassone-Corsi P 1998 Impairing follicle-stimulating hormone (FSH) signaling in vivo: targeted disruption of the $\mathrm{FSH}$ receptor leads to aberrant gametogenesis and hormonal imbalance. PNAS 95 13612-13617.

Dissen GA, Hill DF, Costa ME, Ma YJ \& Ojeda SR 1991 Nerve growth factor receptors in the peripubertal rat ovary. Molecular Endocrinology $\mathbf{5}$ 1642-1650.

Dissen GA, Newman Hirshfield A, Malamed S \& Ojeda SR 1995 Expression of neurotrophins and their receptors in the mammalian ovary is developmentally regulated: changes at the time of folliculogenesis. Endocrinology 136 4681-4692.

Dissen GA, Hill DF, Costa ME, Dees WL, Lara HE \& Ojeda SR 1996 A role for trkA nerve growth factor receptors in mammalian ovulation. Endocrinology 137 198-209.

Dissen GA, Romero C, Newman Hirshfield A \& Ojeda SR 2001 Nerve growth factor is required for early follicular development in the mammalian ovary. Endocrinology 142 2078-2086.

Dissen GA, Paredes A, Romero C, Dees WL \& Ojeda SR 2004 Neural and neurotrophic control of ovarian development. In The Ovary, 2 edn, pp 3-23. Eds P Leung \& E Adashi. San Diego, CA: Academic Press.

Ernfors P, Wetmore C, Olson L \& Persson H 1990 Identification of cells in rat brain and peripheral tissues expressing mRNA for members of the nerve growth factor family. Neuron 5 511-526.

Hallböök F, Ibañez CF \& Persson H 1991 Evolutionary studies of the nerve growth factor family reveal a novel member abundantly expressed in Xenopus ovary. Neuron 6 845-858.

Hirshfield AN 1991 Development of follicles in the mammalian ovary. International Review of Cytology 124 43-101.
Hirshfield AN \& DeSanti AM 1995 Patterns of ovarian cell proliferation in rats during the embryonic period and the first three weeks postpartum. Biology of Reproduction 53 1208-1221.

Kerr B, Paredes A, Garcia-Rudaz C, De la Chesnaye E, Dissen GA \& Ojeda SR 2006 Neurotrophins acting via TrkB receptors use the Notch-Jagged1 cell-cell communication pathway to facilitate early ovarian development. Proceedings of the Endocrine Society, 88th Annual Meeting, Boston, MA, USA. Abstract OR38-1.

Klein R, Parada LF, Coulier F \& Barbacid M 1989 trkB a novel tyrosine protein kinase receptor expressed during mouse neural development. EMBO Journal 8 3701-3709.

Lamballe F, Klein R \& Barbacid M 1991 trkC a new member of the trk family of tyrosine protein kinases, is a receptor for neurotrophin-3. Cell $\mathbf{6 6}$ 967-979.

Lara HE, Hill DF, Katz KH \& Ojeda SR 1990 The gene encoding nerve growth factor is expressed in the immature rat ovary: effect of denervation and hormonal treatment. Endocrinology 126 357-363.

Lee K-F, Li E, Huber LJ, Landis SC, Sharpe AH, Chao MV \& Jaenisch R 1992 Targeted mutation of the gene encoding the low affinity NGF receptor p75 leads to deficits in the peripheral sensory nervous system. Cell 69 $737-749$

Liebl DJ, Klesse LJ, Tessarollo L, Wohlman T \& Parada LF 2000 Loss of brain-derived neurotrophic factor-dependent neural crest-derived sensory neurons in neurotrophin-4 mutant mice. PNAS 97 2297-2302.

McClellan KA, Gosden R \& Taketo T 2003 Continuous loss of oocytes throughout meiotic prophase in the normal mouse ovary. Developmental Biology 258 334-348.

Morse SM, Shaw G \& Larner SF 2006 Concurrent mRNA and protein extraction from the same experimental sample using a commercially available column-based RNA preparation kit. BioTechniques 4054 , 56, 58.

Paredes A, Romero C, Dissen GA, DeChiara TM, Reichardt L, Cornea A, Ojeda SR \& Xu B 2004 TrkB receptors are required for follicular growth and oocyte survival in the mammalian ovary. Developmental Biology 267 430-449.

Paul CE, Vereker E, Dickson KM \& Barker PA 2004 A pro-apoptotic fragment of the p75 neurotrophin receptor is expressed in p75NTRExonIV null mice. Journal of Neuroscience 24 1917-1923.

Peters H 1969 The development of the mouse ovary from birth to maturity. Acta Endocrinologica 62 98-116.

Rajah R, Glaser EM \& Hirshfield AN 1992 The changing architecture of the neonatal rat ovary during histogenesis. Developmental Dynamics 194 177-192.

Romero C, Paredes A, Dissen GA \& Ojeda SR 2002 Nerve growth factor induces the expression of functional FSH receptors in newly formed follicles of the rat ovary. Endocrinology 143 1485-1494.

von Schack D, Casademunt E, Schweigreiter R, Meyer M, Bibel M \& Dechant G 2001 Complete ablation of the neurotrophin receptor p75NTR causes defects both in the nervous and the vascular system. Nature Neuroscience 4 977-978.

Sicinski P, Donaher JL, Geng Y, Parker SB, Gardner H, Park MY, Robker RL, Richards JS, McGinnis LK, Biggers JD et al. 1996 Cyclin D2 is an FSH-responsive gene involved in gonadal cell proliferation and oncogenesis. Nature 384 470-474.

Spears N, Molinek MD, Robinson LL, Fulton N, Cameron H, Shimoda K, Telfer EE, Anderson RA \& Price DJ 2003 The role of neurotrophin receptors in female germ-cell survival in mouse and human. Development 130 5481-5491.

Tolosa JM, Schjenken JE, Civiti TD, Clifton VL \& Smith R 2007 Column-based method to simultaneously extract DNA, RNA, and proteins from the same sample. BioTechniques 43 799-804.

Zhu G-Z, Miller BJ, Boucheix C, Rubinstein E, Liu CC, Hynes RO, Myles DG \& Primakoff P 2002 Residues SFQ (173-175) in the large extracellular loop of CD9 are required for gamete fusion. Development 129 1995-2002.

Received 12 November 2008

First decision 16 January 2009

Revised manuscript received 30 March 2009

Accepted 7 April 2009 